\title{
Analysis of Wood Structural Changes under Thermal Radiation
}

\author{
D. K. Shen, S. Gu, * K. H. Luo, and A. V. Bridgwater \\ School of Engineering Science, University of Southampton, Highfield, Southampton SO17 1BJ, United \\ Kingdom, and School of Engineering and Applied Science, Aston University, Aston Triangle, Birmingham \\ B4 7ET, United Kingdom
}

Received October 11, 2008. Revised Manuscript Received January 4, 2009

\begin{abstract}
Experiment is carried out to observe the structural changes of cellulosic materials exposed to thermal radiation. To quantitatively analyze the results of pyrolyzed wood samples, simple geometric models are proposed to include both shrinkage and cracking. The shrinkage factors consider each direction of three-dimensional geometry, as well as the overall volume. The cracking coefficients include the depth and volume of cracks. The structural changes of the solid are characterized during the pyrolysis by varying the external heat flux and the species of wood. Formulas for calculation of crack depths are derived based on the experimental results for softwood and hardwood. The expressions of cracking can be incorporated into pyrolysis models to include the effects of cracks on heat and mass transfer during wood pyrolysis.
\end{abstract}

\section{Introduction}

Wood and other cellulosic materials are widely used in buildings and increasingly utilized as renewable sources of energy. Much research efforts have been given to underpin the kinetics of thermal decomposition, whereas there is still lack of quantitative data or accurate models to describe the structural change of the solid fuel during biomass degradation. Some experiments conducted for large biomass particles have shown significant shrinkage of the char layer as the pryolysis propagates through the solid. ${ }^{1}$ Additional experimental studies implemented by Roberts ${ }^{2}$ and Spearpoint ${ }^{3}$ describe the structural changes of the large biomass solid in the presence of shrinkage and surface cracks at pyrolysis temperatures above $300{ }^{\circ} \mathrm{C}$. Most data concerning the shrinkage of large biomass particles is obtained by the Forest Products Laboratory; ${ }^{4}$ however, the shrinkage factor provided is in either one dimension or global volume of the solid. Davidsson et al. ${ }^{5}$ conducted a set of experiments to investigate both the volume shrinkage of wood particles and the shrinkage in three dimensions. It is elucidated that the volume shrinkage is found to be $45-70 \%$ and the shrinkages are $5-25,15-40,25-40 \%$ for the longitudinal, radial, and tangential directions, respectively. The occurrence of shrinkage is believed to be attributed to water loss, rearrangement of chemical bonds, and coalescence of graphite nuclei within the solid structure. ${ }^{6}$

The structural changes of the solid in forms of shrinkage and cracking affect the pyrolysis in several ways. The char density increases as a result of chemical rearrangement, and the shortened distance across the pyrolysis region changes the temperature profile of the solid. ${ }^{6}$ The cracks from the surface

* To whom correspondence should be addressed. E-mail: s.gu@soton.ac.uk, ds1t07@soton.ac.uk.

(1) Chan, W. R.; Kelbon, M.; Krieger, B. B. Fuel 1985, 64, 1505-1513.

(2) Roberts, A. F. Combust. Sci. Technol. 1971, 4, 71-83.

(3) Spearpoint, M. J. NIST GCR 99-975: 1999.

(4) Forest Products Laboratory, Wood handbook: Wood as an engineering material agricultural handbook 72; Department of Agriculture: Washington, DC, 1987.

(5) Davidsson, K. O.; Pettersson, J. B. C. Fuel 2002, 81, 263-270.

(6) Hagge, M. J.; Bryden, K. M. Chem. Eng. Sci. 2002, 57, 2811-2823. allow heat to reach the inside of the solid much faster. It is also reported that the shrinkage contributes to reduction of the pyrolysis time and to change of the product yield (e.g., increase of tar and reduction of volatiles). ${ }^{7}$ Therefore, it is important to consider the structural changes such as shrinkage and cracking in biomass decomposition models for more accurate prediction. Shrinkage models are available today and generally in the categories of either uniform shrinkage 6,8 or multidimensional shrinkage. ${ }^{5,7,9-12}$ Although the uniform shrinkage models can be easily coupled with pyrolysis models, the assumption of identical change in all directions limits their predictability. Multidimensional shrinkage is more accurate by considering the variation of spatial coordinates; however, most shrinkage factors from existing models are not based on experimental data, and their results are debatable.

In addition to the limitation about current shrinkage models, literature about quantitative analysis on cracking phenomena of solid fuels is scarce. In this paper, experiment is carried out to investigate the structural changes in forms of both shrinkage (three-dimensional shrinkages) and cracking (volume and depth of cracks) of the solid fuel when wood samples are exposed to thermal radiation. Effects of heat fluxes, wood species, and heating time on the shrinkage factors and cracking coefficients are examined. Empirical functions are derived to calculate the cracking depth for softwood and hardwood pyrolysis.

\section{Experiment and Structure Models}

The experimental system described in ref 13 is used for this investigation. Three types of wood, namely pine-softwood, birch-

(7) Larfeldt, J.; Leckner, B.; Melaaen, M. C. Fuel 2000, 79, 1637-1643.

(8) Tran, H. C.; White, R. H. Fire Mater. 1992, 16, 197-206.

(9) Parker, W. J. Prediction of heat release rate in wood. Ph.D. Thesis, George Washington University, 1988.

(10) Blasi, C. D. Chem. Eng. Sci. 1996, 51, 1121-1132.

(11) Bellais, M.; Davidsson, K. O.; Liliedahl, T.; Sjostrom, K.; Pettersson, J. B. C. Fuel 2003, 82, 1541-1548.

(12) Babu, B. V.; Chaurasia, A. S. Chem. Eng. Sci. 2004, 59, 19992012.

(13) Shen, D. K.; Fang, M. X.; Luo, Z. Y.; Cen, K. F. Fire Saf. J. 2007, $42,210-217$. 
Table 1. Properties of the Wood Samples Tested in the Experiment

\begin{tabular}{lccr}
\hline & pine & birch & floor board \\
\hline density $\left(\mathrm{kg} / \mathrm{m}^{3}\right)$ & 396 & 796 & 844 \\
low heating value $(\mathrm{kJ} / \mathrm{kg})$ & 18129 & 16863 & 18872 \\
& Proximate & Analysis (As Received, \%) & \\
moisture & 9.54 & 11.39 & 7.78 \\
volatiles & 74.21 & 74.36 & 76.84 \\
fixed carbon & 13.06 & 13.49 & 15.16 \\
ash & 3.19 & 0.76 & 0.22 \\
& & & \\
carbon & Ultimate Analysis (As Received, \%) & 46.37 \\
hydrogen & 41.89 & 44.41 & 5.13 \\
nitrogen & 4.5 & 3.48 & 0.28 \\
oxygen & 0.22 & 0.27 & 40.29 \\
sulfur & 40.19 & 36.65 & \\
& 0.15 & & \\
hemicellulose & Constituent Analysis & $(\%)$ & \\
cellulose & 15.37 & 24.79 & \\
lignin & 52.10 & 56.47 & \\
inorganic materials & 27.45 & 12.17 & \\
& 5.08 & 6.57 &
\end{tabular}

hardwood, and floorboard-artificial wood are used in the experiment, and their properties are shown in Table 1. The wood samples are produced with the surface area of $100 \mathrm{~mm}$ by $100 \mathrm{~mm}$ and thickness of $15 \mathrm{~mm}$. The samples are placed under atmospheric condition with uniform thermal intensities from 15 to $60 \mathrm{~kW} / \mathrm{m}^{2}$. The temperature distribution, mass loss, ignition time, and pyrolysis duration of the solid are recorded during the experiment. The details of measurement procedure can be found in ref 13 .

The structural changes of the solid samples during thermal decomposition are illustrated in Figure 1. To avoid confusion, the longitudinal, radial, and tangential directions in this paper refer to the directions along, across, or into the depth of the grain, respectively. It is apparent from the decomposed samples that the shrinkages develop differently in each direction while the cracks are not evenly developed in longitudinal and radial directions. Besides, the degree of shrinkage and cracking of the solid vary with the heat flux and heating time. On the basis of those structural characters, a geometry model is developed to quantitatively represent the shrinkage and cracking as shown in Figure 2. The model assumes that the cracks in the same direction share the same characteristics (width, length, and depth). The shrinkage factors and cracking coefficients are defined as follows.

The initial volume of the solid is

$$
V_{0}=L_{p 0} \times L_{a 0} \times D_{0}
$$

The volume of the shrinkaged solid with cracks is described as

$$
V=\left(L_{p 1}+L_{p 2}\right) / 2 \times L_{a} \times D
$$

while the total volume for the cracks is defined as

$$
V_{w}=\left(N_{p} \times L_{p 1} \times W_{p}+N_{a} \times L_{a} \times W_{a}\right) / 2 \times d_{w}
$$

The volume shrinkage of the solid is defined as

$$
\eta_{v}=\left(V-V_{w}\right) / V_{0}
$$

The shrinkages in different directions are given as

$$
\eta_{a}=L_{a} / L_{a 0} ; \quad \eta_{p}=L_{p 1} / L_{p 0} ; \quad \eta_{D}=D / D_{0}
$$

The cracking coefficients for the volume and depth at a certain stage are defined as

$$
f_{v}=V_{c} / V_{0} ; \quad f_{D}=d_{w} / D_{0}
$$

\section{Results and Discussion}

The decomposed samples are measured by a vernier caliper, and the structural changes are calculated with the geometric models described above. The inner structures of the solid are obtained with razor blade cutting and characterized by a scanning electron microscope (SEM). The results of thermal decomposition with emphasis on structural changes are discussed in this section.

3.1. Phenomena of Structural Changes. Global Shrinkage. The results from the pine samples under heat flux of $15 \mathrm{~kW} / \mathrm{m}^{2}$ are shown in Figure 3. Despite the variation in each direction, the steady development of shrinkages during the heating period illustrated in Figure $3 \mathrm{a}$ is consistent with the theory. It is known that the biomass is mainly composed of the following: hemicellulose (the thermally least stable component decomposing at $220^{\circ} \mathrm{C}$ ), lignin (decomposing between 200 to $500{ }^{\circ} \mathrm{C}$ ), and cellulose (decomposing start at around $280{ }^{\circ} \mathrm{C}$ ). ${ }^{14}$ The three components generate different products including gases, tars, and charcoal as a result of the corresponding chemical reactions for the different polymer chains during the pyrolysis. ${ }^{15}$ It is known that most of the charcoal in the pyrolyzed samples is formed from the decomposition of hemicellulose and lignin, which to some extent determines the degree of the structural changes (both shrinkage and cracks). It is described by Davidsson and Pettersson ${ }^{5}$ that while the heating is fast enough for char forming reactions of hemicellulose and lignin, the cleavage of cellulose chains would indeed affect the shrinkage of the solid. The shrinkage of the solid biomass during the pyrolysis can be described in microscopic (e.g., cracks) and molecular levels (e.g., cleavage of the polymer chains). The global shrinkage is mainly caused by the scission and contraction of the various polymer chains which consist of the three main components of wood and the coalescence of graphite nuclei within the solid structure. ${ }^{6}$ The decomposition of those components is enhanced when the temperature of the solid increases during the heating period, and consequently, the shrinkage continues with the time.

Shrinkage Variation. The variation of shrinkage in different directions is evident in Figure 3a, that is, a much smaller reduction in the longitudinal direction. The phenomenon has also been observed by other researchers. ${ }^{5,7,16}$ The microstructure of the solid could explain such phenomenon to some extent. The microstructure of the charcoal of pine after the pyrolysis shown in Figure 4 shows that the heterogeneous cell structure consists of the arrays of carbon skeleton vessels arranged along the longitudinal direction. This confirms the existing finding that the wood has a similar microstructure composed of the cellulose microfibrils covered by hemicellulose-lignin matrix. ${ }^{17}$ The cell structure of wood can be schematically shown in Figure 5. It is apparent that the freedom in the longitudinal direction for the cell structure is less than in other directions, which could influence the shrinkage of the solid in a similar fashion. More hemicellulose and lignin deposited along the tangential and radial directions in the cellulose microfibrils give easy evolution of the volatiles; therefore, structure change starts earlier and develops faster in those directions.

Moreover, the reaction mechanisms of decomposition may also contribute to the variation of shrinkage. The three main components of wood decompose at different temperatures. A time-dependent temperature profile close to the surface of the solid will indicate the decomposition period for each component. Thermocouples are placed in the pine samples at various depths, namely, $1 \mathrm{~mm}, 6 \mathrm{~mm}$, and $13 \mathrm{~mm}$ from the heating surface.

(14) Antal, M. J.; Varhegyi, G. Ind. Eng. Chem. Res. 1995, 34, 703717.

(15) Hosoya, T.; Kawamoto, H.; Saka, S. J. Anal. Appl. Pyrolysis 2007, $78,328-336$.

(16) Connoer, M. A.; J. Ward., V. D.

(17) Hosoya, T.; Kawamoto, H.; Saka, S. J. Anal. Appl. Pyrolysis 2007, 80, 118-125. 


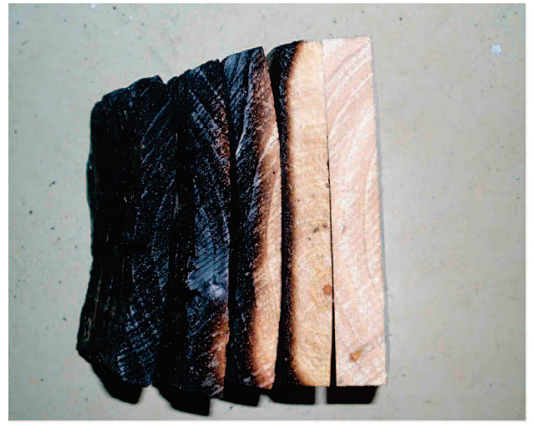

Pine, $15 \mathrm{~kW} / \mathrm{m}^{2}(1020-0 \mathrm{~s})$

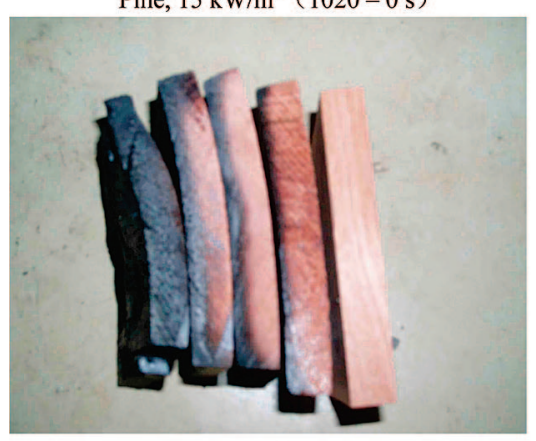

Birch, $15 \mathrm{~kW} / \mathrm{m}^{2}(1140-0 \mathrm{~s})$

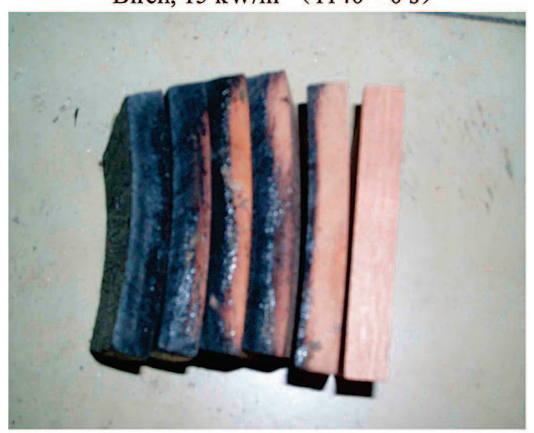

Floor board, $15 \mathrm{~kW} / \mathrm{m}^{2}(1380-0 \mathrm{~s})$

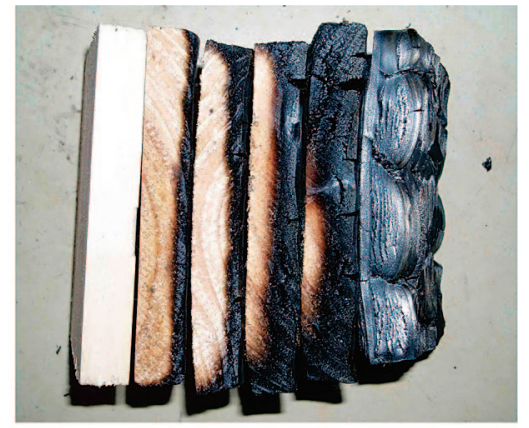

Pine, $50 \mathrm{~kW} / \mathrm{m}^{2}(0-420 \mathrm{~s})$

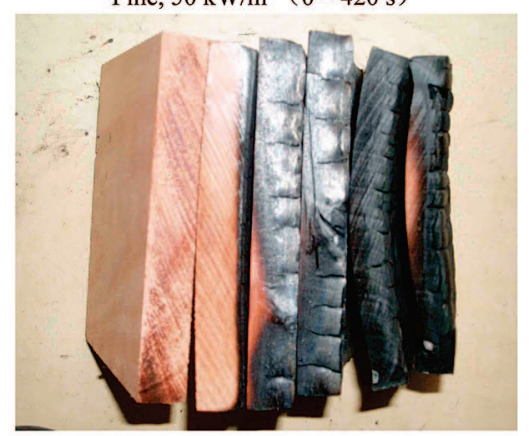

Birch, $50 \mathrm{~kW} / \mathrm{m}^{2}(0-420 \mathrm{~s})$

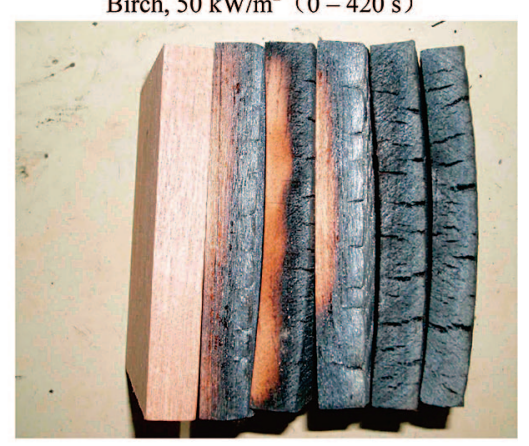

Floor board, $50 \mathrm{~kW} / \mathrm{m}^{2}(0-420 \mathrm{~s})$

Figure 1. Shrinkage and cracking of solid samples during pyrolysis.

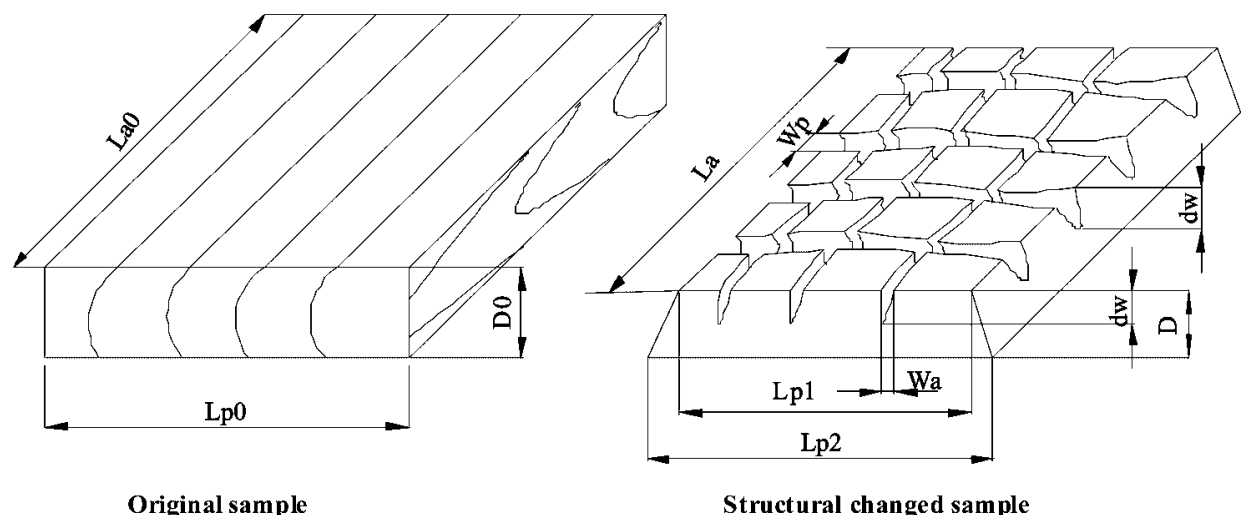

Figure 2. Geometry model for the structural changes of decomposed solid.

The temperature evolution for $1 \mathrm{~mm}$ depth below the surface shown in Figure 3c demonstrates that the solid temperature reaches $280{ }^{\circ} \mathrm{C}$ (decomposition temperature for cellulose) around $400 \mathrm{~s}$. At the same time, in Figure 3a, the shrinkage in the longitudinal direction becomes noticeable. In the same way, it is found that the solid temperature reaches $220^{\circ} \mathrm{C}$ (decomposition temperature for hemicellulose and lignin) about $100 \mathrm{~s}$, when the shrinkages in the radial and tangential directions start. These experimental results confirm the finding in ref 5 that the shrinkage in the longitudinal direction is mainly ascribed to contraction of the cellulose chain because of the oxygen depletion, while the shrinkage in the radial and tangential directions are mainly due to devolatilization of hemicellulose and lignin.

Crack Development. The development of cracks also increases with the heating time in terms of both depth and total volume as shown in Figure $3 \mathrm{~b}$. The mechanism of cracking is contributed by the disruption of the walls between two adjacent wood cells and the breakup of chemical bonds among the carbon 


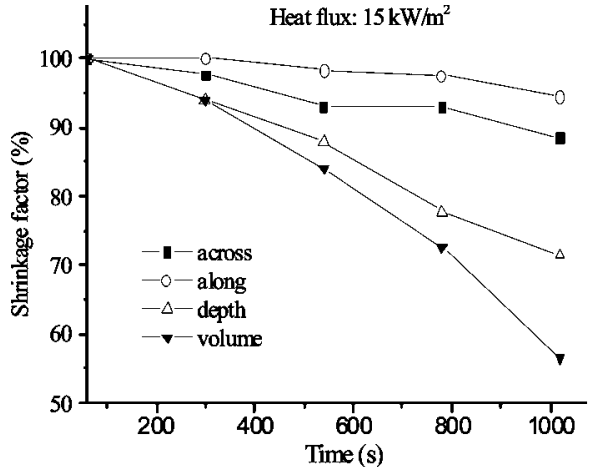

(a)

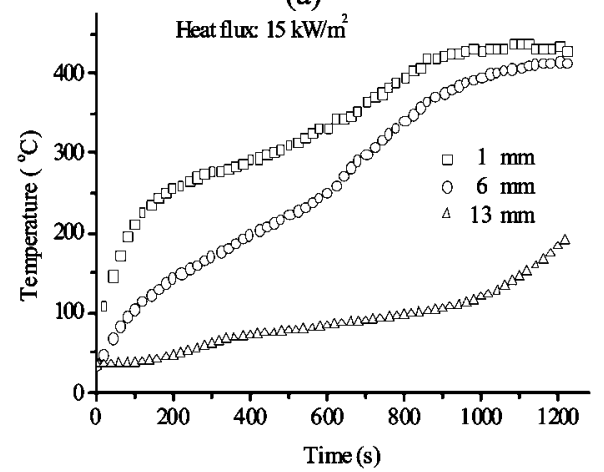

(c)

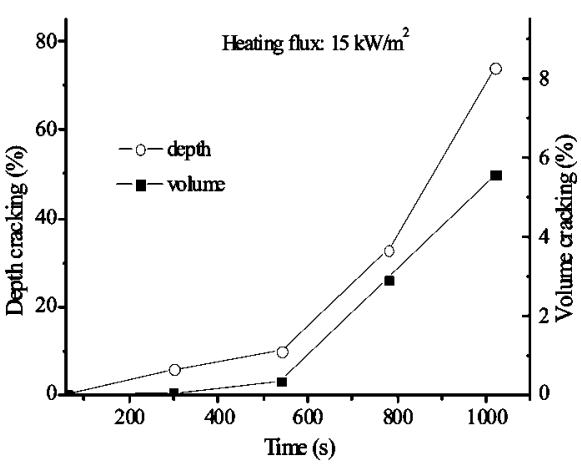

(b)

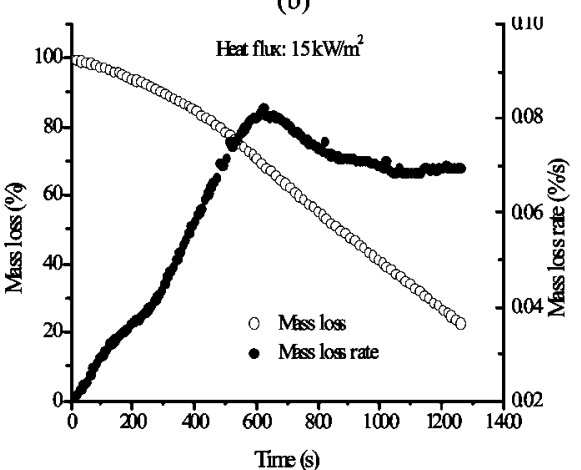

(d)

Figure 3. Characteristics of the pyrolyzed pine samples: (a) the shrinkage factors; (b) the cracking coefficients; (c) the temperature distribution at different depths; (d) the mass loss.

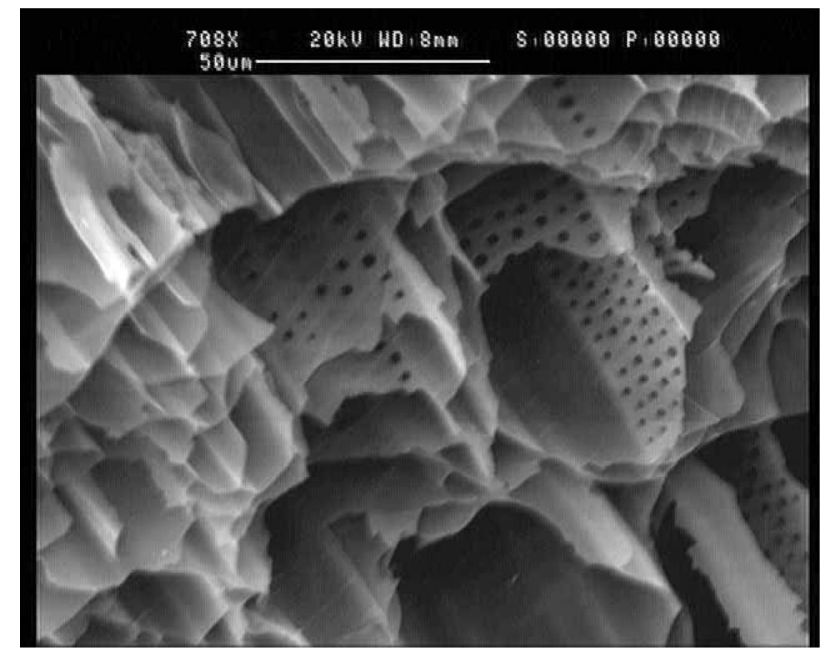

Figure 4. Scanning electron microscopic image of the pyrolyzed pine sample under the heat flux of $15 \mathrm{~kW} / \mathrm{m}^{2}$.

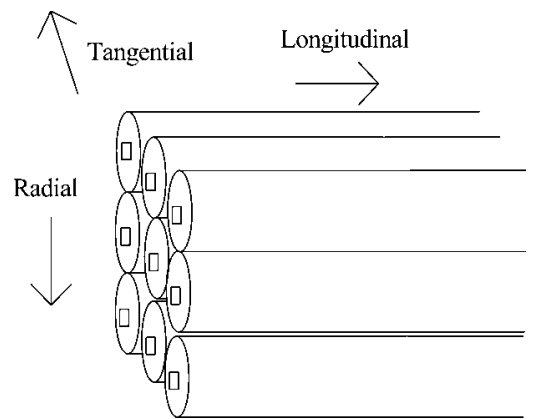

Figure 5. Schematic of the microfibril structure of wood.

atoms caused by the scission of the polymer chains during the pyrolysis. The growth of cracks is also associated with the rearrangement of the charcoal structure while the cell vessels are affected by the temperature and evolution of gases. The above chemical and physical factors related to crack development are enhanced at higher temperature, which intensifies cracking.

The temperature profiles at various depths inside the wood samples under the heat flux of $15 \mathrm{~kW} / \mathrm{m}^{2}$ are shown in Figure $3 c$. During the monitoring process of crack growths in the experiment, we found that the cracks reach $1 \mathrm{~mm}$ depth at $300 \mathrm{~s}$, $6 \mathrm{~mm}$ at $600 \mathrm{~s}$, and $13 \mathrm{~mm}$ at $1100 \mathrm{~s}$. Once the cracks reach there, we can see from the temperature plots of $1,6,13 \mathrm{~mm}$ depths that a sharper increase of temperature appears in Figure 3c. In comparison to Figure 3d, the rate of mass loss of the solid increases sharply at $300 \mathrm{~s}$ when the $1 \mathrm{~mm}$ cracks are generated. It is expected that the cracks enable heat to penetrate inside of the solid more easily, accelerating the decomposition and release of volatiles. However, further investigation in Figure $3 \mathrm{~d}$ reveals that no noticeable increase of mass loss rate appears at $600 \mathrm{~s}$ when the $6 \mathrm{~mm}$ cracks are formed, and the mass loss rate is in a steady decline later on. A possible explanation could be that the solid is gradually covered by the char layers which resist easy heat propagation and escape of volatiles from the solid, and as a result, the mass loss rate starts to decline shortly after 600s.

3.2. Shrinkage Factors. Effect of Heat Flux. Different heat fluxes are applied to the wood samples, and the results from the pine are shown in Figure 6. It is found that the shrinkage develops faster in each direction and for the overall volume when the heat flux is increased from 15 to $30 \mathrm{~kW} / \mathrm{m}^{2}$. At low heating such as $15 \mathrm{~kW} / \mathrm{m}^{2}$, char is produced mainly from the hemicellulose and partly from lignin to form a rigid carbon structure around the microfibril to resist the shrinkage of the solid. On the other hand, at the high heat flux of $30 \mathrm{~kW} / \mathrm{m}^{2}$, heat transfer is fast enough for the char forming reactions of hemicellulose and lignin to be overlapped with chain scission and contraction of cellulose, which leads to unstable char 


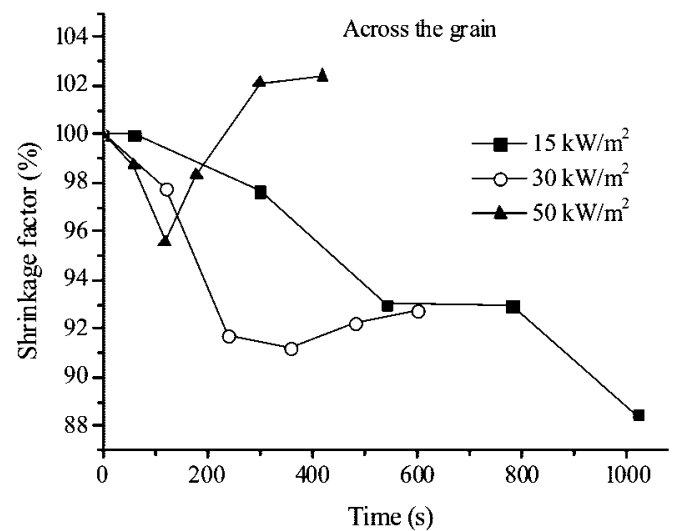

(a)

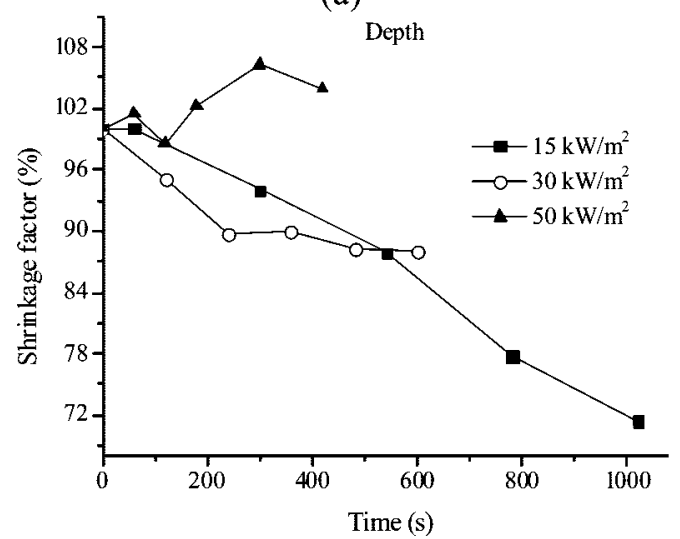

(c)

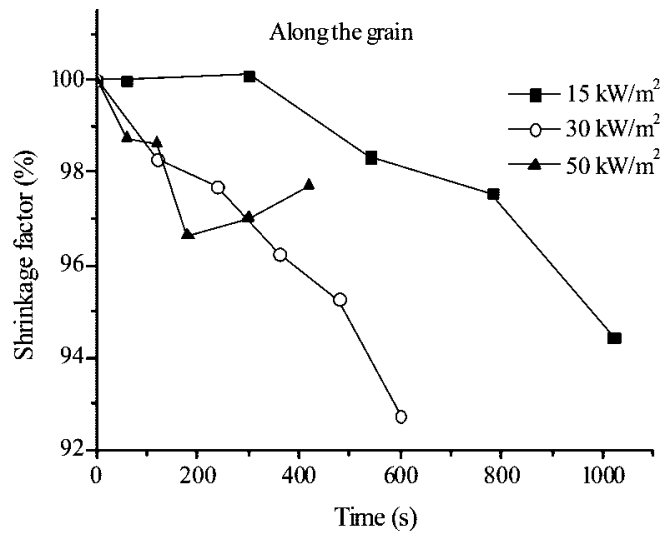

(b)

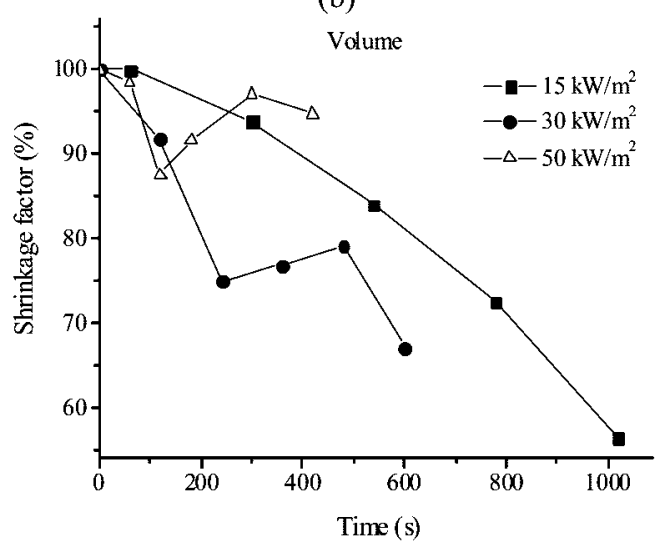

(d)

Figure 6. Shrinkage factors of pine samples under different heat fluxes: (a) across the grain; (b) along the grain; (c) in the depth; (d) volume.

structure, fast coalescence of carbon nuclei, and larger structural changes in each dimension of the solid. When the heat flux is further increased to $50 \mathrm{~kW} / \mathrm{m}^{2}$, the shrinkage factors become more unpredictable, and the solid even swells at a certain stage. The study by Davidsson and Pettersson ${ }^{5}$ has also shown less shrinkage in the radial and tangential directions and for the total volume of the solid. However, the swelling structural changes of the solid observed in our experiment have not been reported before.

Effect of Species. The results from the birch samples at the same heat fluxes as the pine are shown in Figure 7. The birch samples show less shrinkage than the pine under the same heat flux. It is known that high content of hemicellulose contributes to a rigid char structure in the pyrolyzed solid. Thus, it is more difficult for the birch to change the structure as it contains more hemicellulose than the pine (Table 1), leading to a smaller shrinkage for the birch. A comparison between Figures 6 and 7 also shows that the shrinkage continues to increase for the birch as the heat flux rises to $50 \mathrm{~kW} / \mathrm{m}^{2}$ without noticeable appearance of swelling. This could be explained by the fraction of components and physical properties of the solid. The rigid char structure in the pyrolyzed solid resulted from the decomposition of the high content of hemicellulose (here birch contains more hemicellulose than pine), and the generation of gases during the pyrolysis of hard wood (birch) is slower than that of softwood (pine) because of the physical properties. ${ }^{13}$ The rapid release of gases for pine under the high heat flux builds up pressure inside the solid, and the formed char structure is more easily stretched and compressed at the high temperature. Therefore, the increased gas flow and higher pressure give rise to higher mechanical stress which contributes to the greater shrinkage (or cracking as described below) of the solid. The swelling phenomenon for the pine occurs at the condition when the shrinkage is overwhelmed by the cracking of the solid.

3.3. Cracking Coefficients. Cracking Mechanism. The cracking coefficients for the pine and birch wood are plotted as a function of time in Figure 8. It is apparent that the general trends for both species are similar, the cracking intensifies both in depth and volume during the heating period, and the increase of heat flux from 15 to $50 \mathrm{~kW} / \mathrm{m}^{2}$ also accelerates the cracking several times over. It is believed that the cracking of solid fuels is due to the disruption of the cell walls between two adjacent wood cells and rearrangement of the chemical bond of the carbon atoms. The process intensifies as the temperature of the solid increases, and gives rise to larger cracks as heating continues. The results in Figure 8c quantitatively show that the cracking coefficients increase with heat flux, for example, the cracks for the birch wood start at $480 \mathrm{~s}$ under $15 \mathrm{~kW} / \mathrm{m}^{2}, 120 \mathrm{~s}$ under 30 $\mathrm{kW} / \mathrm{m}^{2}$, and $60 \mathrm{~s}$ under $50 \mathrm{~kW} / \mathrm{m}^{2}$. The acceleration is due to the high temperature gradient of the solid under high heat flux, and hence the gases are rapidly evolved to accumulate the pressure within the solid during the pyrolysis. The afflux of gases and subsequent build-up of pressure increase the mechanical stress for the structural changes in forms of cracks (or shrinkage as described above). It is evident that higher heat flux gives rise to earlier start of cracking and larger cracks eventually. Between hard and soft woods, the pine samples start to crack earlier than the birch, owing to the different physical properties and component fractions as described in the shrinkage results.

Relation to Mass Loss. The relationship between the cracking coefficient and mass loss rate of the solid fuels are shown in Figures 9 and 10. Various samples are used, and the sequence of initial cracking is pine, birch, and floor board. The largest cracks are shown in the pine samples because of high thermal 


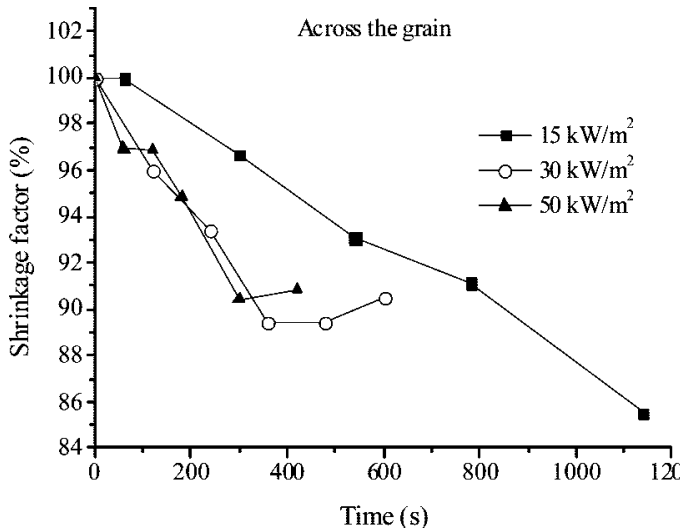

(a)

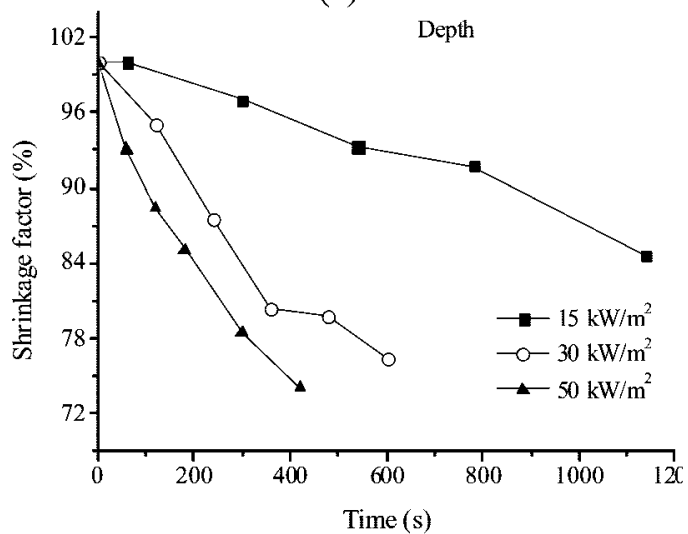

(c)

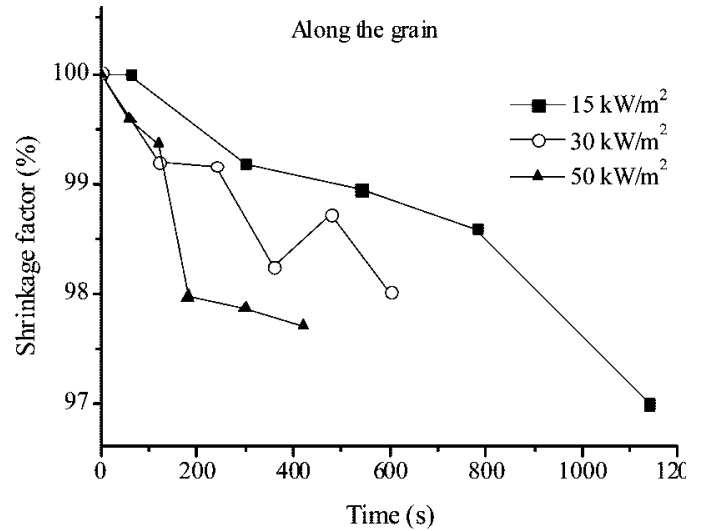

(b)

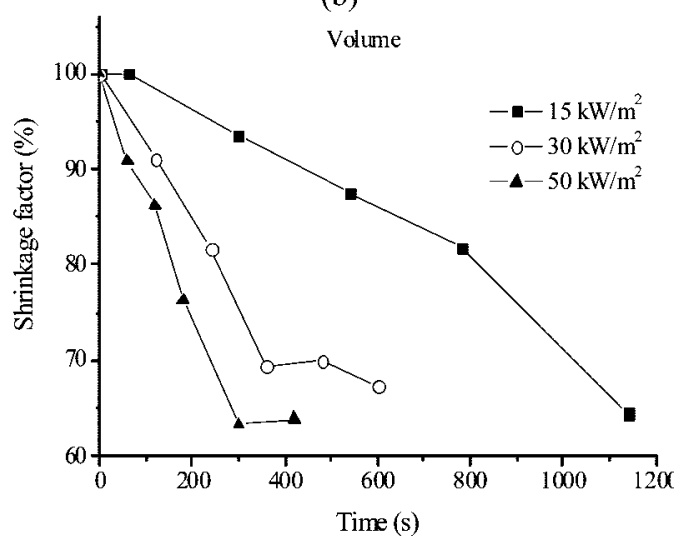

(d)

Figure 7. Shrinkage factors of birch samples under different heat fluxes: (a) across the grain; (b) along the grain; (c) in the depth; (d) volume.

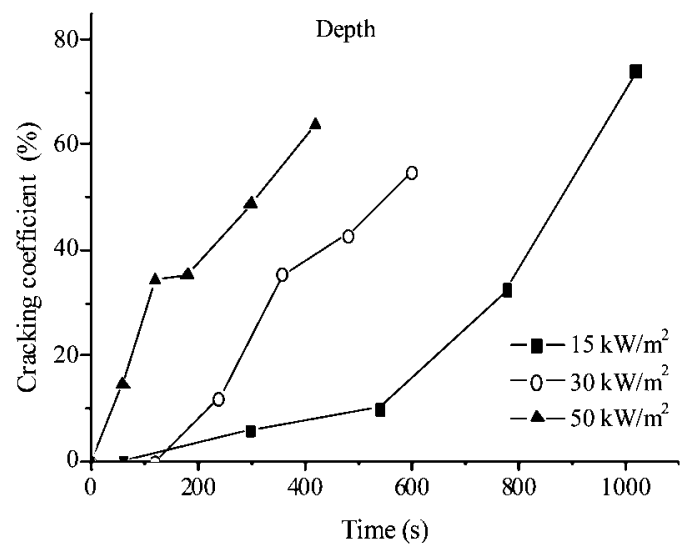

(a)

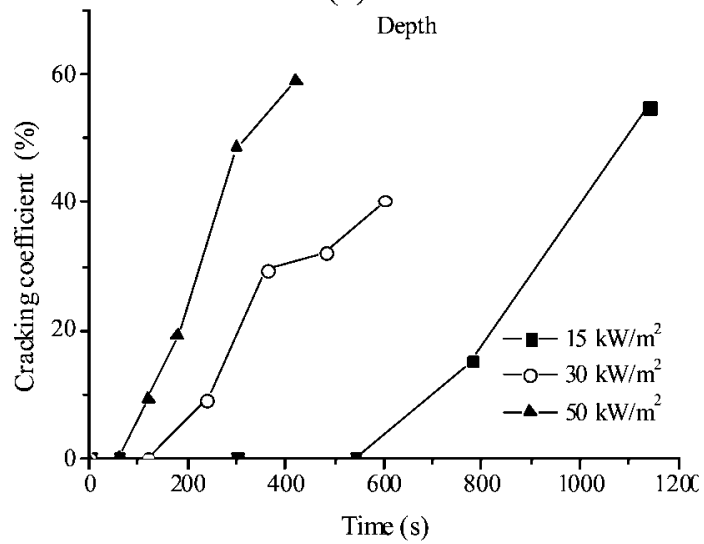

(c)

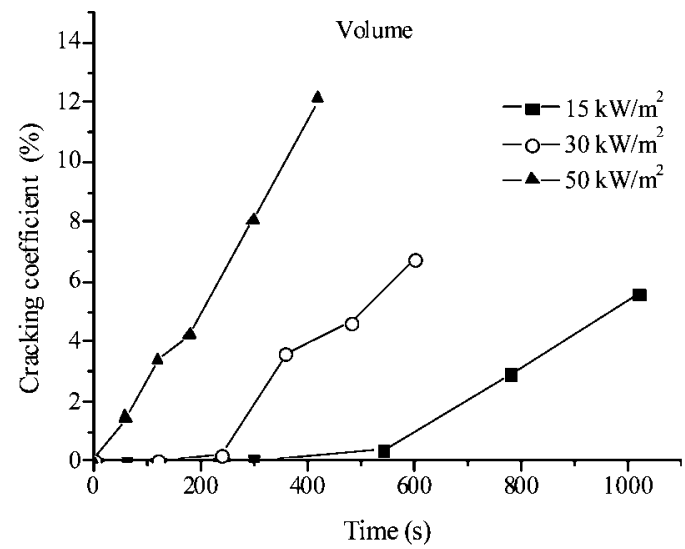

(b)

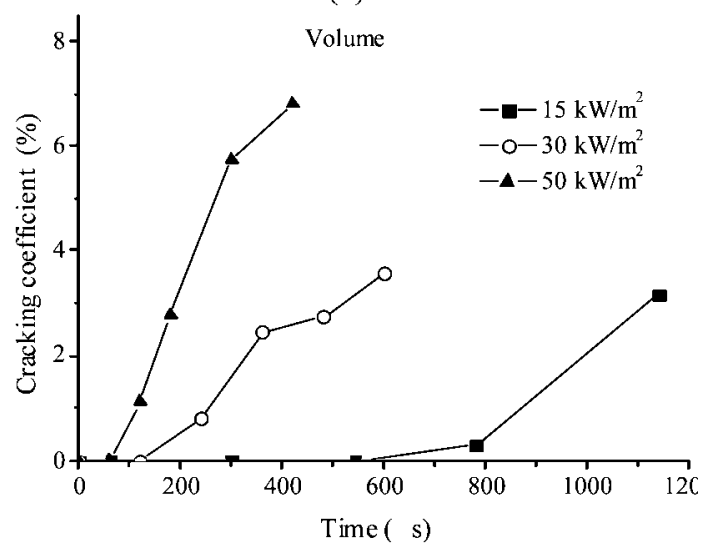

(d)

Figure 8. Cracking coefficients of pine and birch samples under different heat fluxes: (a) depth coefficient for pine; (b) volume coefficient for pine; (c) depth coefficient for birch; (d) volume coefficient for birch. 


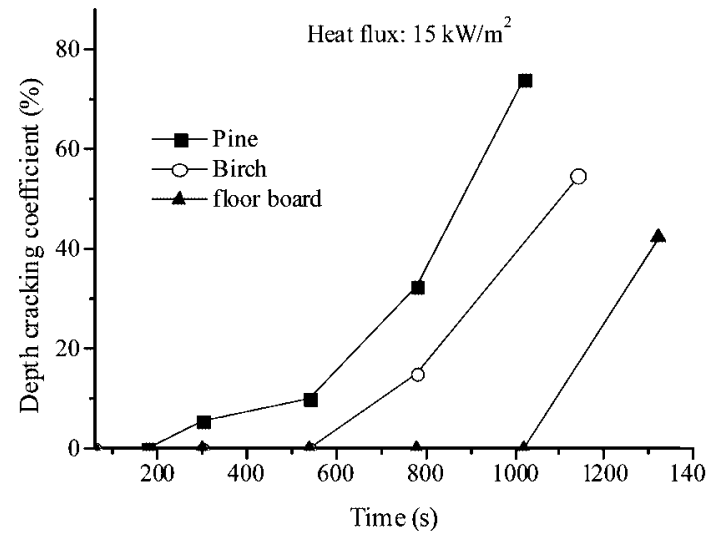

(a)

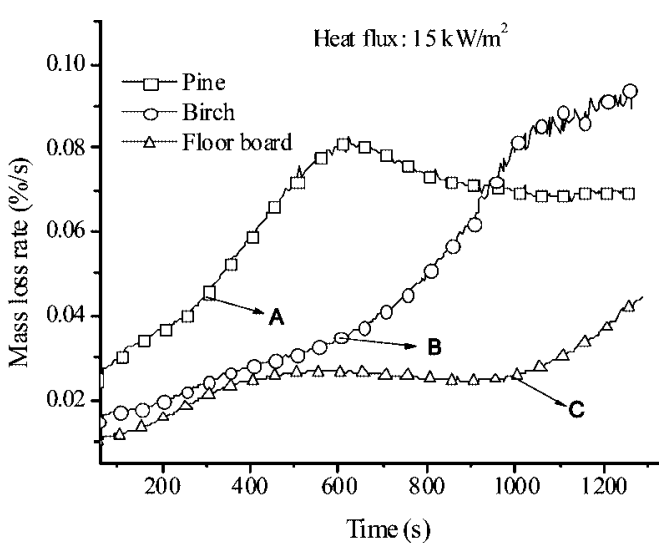

(b)

Figure 9. Cracking coefficient and mass loss rate of wood under the heat flux of $15 \mathrm{~kW} / \mathrm{m}^{2}$ : (a) volume cracking coefficient; (b) mass loss rate.

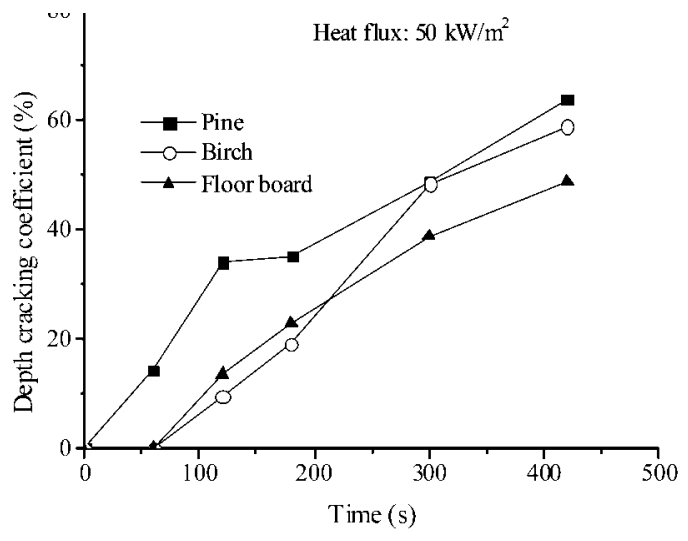

(a)

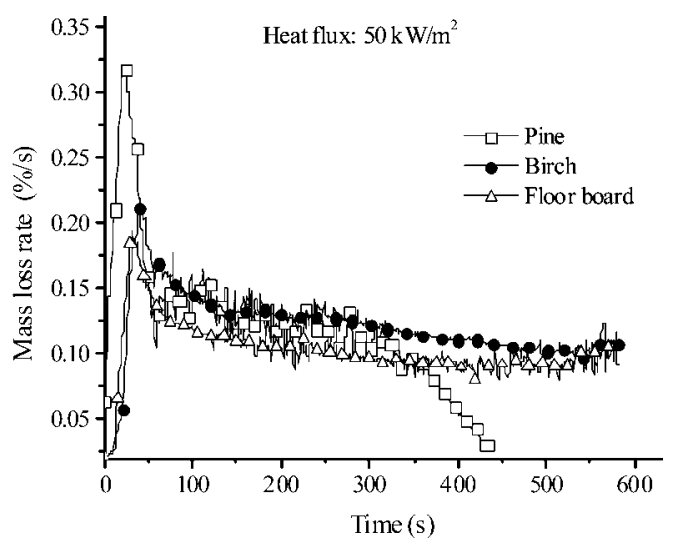

(b)

Figure 10. Cracking coefficient and mass loss rate of wood under the heat flux of $50 \mathrm{~kW} / \mathrm{m}^{2}$ : (a) volume cracking coefficient; (b) mass loss rate.

conductivity which promotes char conversion during pyrolysis. Cracks start to appear on the surface of the pine at around $240 \mathrm{~s}$ (Figure 9a), and corresponding to that, the mass loss rate accelerates from $250 \mathrm{~s}$ (Figure 9b). It is apparent that the cracks enable thermal radiation to penetrate deeper into the wood, enhance the pyrolysis process, release more volatiles, and subsequently accelerate the mass loss of the solid. Similar mechanism is applied to the birch and floor board samples with much delayed appearance, that is, the birch starts to crack with mass loss acceleration at $600 \mathrm{~s}$ while the floor board begins to crack with mass loss acceleration at $1000 \mathrm{~s}$. When the heat flux is increased from 15 to $50 \mathrm{~kW} / \mathrm{m}^{2}$, the speed of cracking and mass loss rate are accelerated as shown in Figure 10. All the samples are heated up very quickly under such high heat flux (the difference in thermal conductivity becomes insignificant now), char formation and pyrolysis process are accelerated substantially, even burning appears from the fast release of volatiles, all these contribute to the fast appearance of cracks and very similar cracking coefficient among different samples. However, the real extent of cracking from the pine is still higher than the hardwood, considering the swelling from the pine samples. Figure 10b shows that the mass loss for the pine stops around $450 \mathrm{~s}$ while the birch and floor board continue; evidently the hardwood is better to maintain its physical appearance and stability, which is valuable for fire safety applications. Another parameter that can be used to evaluate chemical and physical stability of solid fuels under thermal radiation is pyrolysis duration, which is defined as the period between the start of the heating and the moment when the mass loss rate of the solid

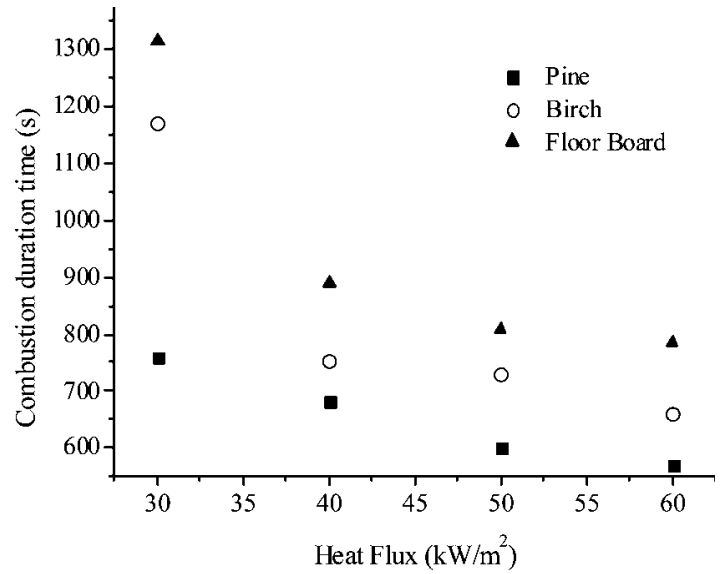

Figure 11. Pyrolysis duration at different heat fluxes.

becomes zero. ${ }^{13}$ A comparison in Figure 11 shows that the pyrolysis duration is decreased with the increased heat flux, and the pine has a shorter period than others which further confirms the previous finding.

Cracking Formulas. The above discussion has highlighted the importance of cracking on the pyrolysis process, in terms of temperature distribution, mass loss rate of solid, and pyrolysis duration. Accurate pyrolysis models need to consider the presence of cracks and their effects on the heat transfer and mass transfer with the solid. In this study, empirical functions of the depth cracking coefficient are derived as follows. 
Softwood

$f_{d}=0.024 \times(H F)^{2}+3.11 \times(H F)+0.0000017 \times t^{2}+$

$$
0.102 \times t-78.52(7)
$$

Hardwood

$$
f_{d}=0.049 \times(H F)^{2}+5.29 \times(H F)-0.000043 \times t^{2}+
$$$$
0.163 \times t-146.59(8)
$$

where $H F$ is the symbol of heat flux with the unit of $\mathrm{kW} / \mathrm{m}^{2}$ and $t$ is the time with a unit of second. The expressions are obtained by the nonlinear fitting of the experimental results, which are taken in a period from 300 to $1020 \mathrm{~s}$ at heating rate of $10 \mathrm{~kW} / \mathrm{m}^{2}$, from 120 to $600 \mathrm{~s}$ at $30 \mathrm{~kW} / \mathrm{m}^{2}$, and from 60 to $420 \mathrm{~s}$ at $50 \mathrm{~kW} / \mathrm{m}^{2}$ for softwood; from 300 to $1380 \mathrm{~s}$ at 10 $\mathrm{kW} / \mathrm{m}^{2}$, from 120 to $600 \mathrm{~s}$ at $30 \mathrm{~kW} / \mathrm{m}^{2}$, and from 60 to $420 \mathrm{~s}$ at $50 \mathrm{~kW} / \mathrm{m}^{2}$ for hardwood. A relative factor is given as 0.9158 for softwood and 0.9128 for hardwood for the functions. The relative factor is a correlation coefficient between the functions and the experimental data. The expressions of cracking coefficients can be incorporated into pyrolysis models to have a quantitative representation of cracks in solid fuels.

\section{Conclusions}

The structural changes, including shrinkage and cracking of the cellulosic materials, are quantitatively studied in this paper. The main conclusions are summarized as follows:

- The shrinkage in the longitudinal direction is found to be less than in other directions because of the microstructure of wood. The main component, cellulose, has longitudinally arranged microfibrils and decomposes later than the other two constituents, hemicellulose and lignin.

- The experiment confirms that the shrinkage in the longitudinal direction is mainly due to contraction of the cellulose chain while the shrinkages in the radial and tangential directions are mainly due to devolatilization of hemicellulose and lignin.

- In general, the development of shrinkage accelerates when heat flux increases. However, swelling appears for the pine samples under the heat flux of $50 \mathrm{~kW} / \mathrm{m}^{2}$, in which case the shrinkage is overwhelmed by the cracking of the solid.

- The development of cracks intensifies when heat flux increases. At low heat flux of $15 \mathrm{~kW} / \mathrm{m}^{2}$, the appearance of cracks on the softwood (pine) is much earlier than the hardwood (birch) while greater cracking is shown for the softwood.

- It is evident that cracks enhance wood pyrolysis and reduce thermal conversion time. The derived formulas for cracking can be readily implemented into existing pyrolysis models to represent the important feature of solid cracking.

\section{Nomenclature}

$L=$ Length of the sample

$D=$ Thickness of the sample

$W=$ Width of crackles

$d=$ Average depth of the cracks

$N=$ Number of crackles per unit

$V=$ Volume

$\eta=$ The shrinkage factor

$f=$ The cracking coefficient

Subscripts

$0=$ The initial stage or original geometry

$1=$ Top surface

$2=$ Bottom surface

$a=$ The direction along the grain

$p=$ The direction across the grain

$v=$ Volume

$D=$ The direction in depth of the grain

$w=$ Crack

EF800873K 